\title{
COMPARAÇÃO ENTRE MÉTODOS PARA O ESTUDO DA ESTABILIDADE DE AGREGADOS EM SOLOS ${ }^{1}$
}

\author{
MARCOS AURÉLIO CAROLINO DE SÁ2, JOSÉ MARIA DE LIMA ${ }^{3}$, MARX LEANDRO NAVES SILVA ${ }^{3}$ \\ e MOACIR DE SOUZA DIAS JUNIOR ${ }^{3}$
}

\begin{abstract}
RESUMO - A estabilidade de agregados constitui-se em importante parâmetro na avaliação do efeito de manejo na agregação de solos. Entretanto, os resultados obtidos pelos diferentes métodos são, às vezes, contrastantes, e alguns desses métodos não permitem a definição do nível de energia envolvido na análise. Objetivou-se, com este estudo, comparar diferentes métodos para determinação da estabilidade de agregados em solos. Agregados de horizontes A e B de Latossolo Roxo (LR) e Terra Roxa Estruturada (TR) foram submetidos a peneiramento úmido, três testes de impacto de gotas, e sonificação a oito níveis de energia ultra-sônica; o primeiro foi comparado aos últimos, que permitem a quantificação da energia aplicada. De modo geral, o LR apresentou agregados mais estáveis do que a TR; a resistência dos agregados a altos níveis de energia ultra-sônica foi relacionada a teores de matéria orgânica, óxidos de ferro e gibbsita. Os resultados de diâmetro médio ponderado e porcentagem de agregados maiores que $2 \mathrm{~mm}$ foram semelhantes nos horizontes A de ambos os solos. O impacto de gotas causou maior desagregação no material de horizonte B do LR, enquanto o material de horizonte B da TR foi mais afetado pelo peneiramento úmido. O nível de $15,88 \mathrm{~J} \mathrm{~mL}^{-1}$ se apresentou, na sonificação, como mais sensível para detectar diferenças entre solos estudados.
\end{abstract}

Termos para indexação: energia, ultra-som, estrutura do solo, estabilização do solo, degradação do solo.

\section{COMPARISON OF METHODS FOR AGGREGATE STABILITY STUDIES IN SOILS}

\begin{abstract}
Aggregate stability is an important parameter to express the effect of management on soil aggregation. However, the results from different methods are sometimes contrasting. Some of them do not express the aggregate stability in terms of level of energy needed for aggregate disruption. The objective of this study was to compare methods for determining soil aggregate stability. Aggregates of Dusky-Red Latossol (DRL) and a Structured Dusky-Red Earth (SDR) were submitted to wet sieving (WS), three water drop tests, and eight levels of sonication energy. Correlation was obtained between the wet sieving and the two other methods. The DRL samples had more stable aggregates than did SDR samples. The resistance to sonication was related to amount of organic matter, Fe oxides and gibbsite. The results of weighted mean diameter and amount of aggregates larger than $2 \mathrm{~mm}$ were the same for the A horizons of both soils. Water dropping caused more disruption on DRL B than on SDR B-horizon material which was more affected by the wet sieving method. The level of $15.88 \mathrm{~J} \mathrm{~mL}^{-1}$ allowed to detect more differences between the soils.
\end{abstract}

Index terms: energy, ultrasonics, soil structure, soil stabilization, soil deterioration.

${ }^{1}$ Aceito para publicação em 29 de dezembro de 1999.

Extraído da dissertação de mestrado apresentada pelo primeiro autor à Universidade Federal de Lavras (UFLA), Lavras, MG.

${ }^{2}$ Eng. Agrôn., M.Sc., Dep. de Ciência do Solo, UFLA, Caixa Postal 37, CEP 37200-000 Lavras, MG.

E-mail: macsa@ufla.br

${ }^{3}$ Eng. Agrôn., D.Sc., Dep. de Ciência do Solo, UFLA. Bolsista do CNPq. E-mail: jmlima@ufla.br, marx@ufla.br, msouzadj@ufla.br

\section{INTRODUÇÃO}

A estabilidade de agregados é um indicador dos processos envolvidos na degradação do solo (Tippkötter, 1994), pois influencia a infiltração, retenção de água, aeração e resistência à penetração de raízes (Santos, 1993; Silva, 1994), selamento e encrostamento superficial (Derpsch et al., 1991), erosão hídrica e eólica (Reichert et al., 
1993; Sun et al., 1995); é também o parâmetro que melhor se correlaciona com a erodibilidade do solo (Angulo, 1983; Egashira et al., 1983).

Com o cultivo, há redução nos teores da matéria orgânica e, conseqüientemente, na estabilidade de agregados, resultando em aumento da proporção relativa de microagregados no solo, cuja estabilidade não é influenciada pelo manejo (Tisdall \& Oades, 1982). A manutenção de agregados estáveis permite minimizar o salpicamento de partículas e é essencial no manejo do solo (Mazurak \& Mosher, 1970). Portanto, o conhecimento de estabilidade de agregados é importante na definição dos indicadores da qualidade do solo.

A avaliação da estabilidade dos agregados em solos depende das forças que ligam as partículas e da natureza e magnitude das forças desagregantes aplicadas nesta avaliação (Beare \& Bruce, 1993). Existem diferenças entre os métodos para avaliação da estabilidade de agregados em solos (Fuller \& Goh, 1992). Por essa razão, a forma de medição da estabilidade dos agregados tem sido motivo de controvérsia, uma vez que a maioria dos métodos se limita a comparações entre solos e tratamentos, e o nível de energia aplicada é arbitrário e desconhecido (Fuller \& Go, 1992). Métodos como o peneiramento úmido de Yoder (1936) e suas modificações (Kemper \& Chepil, 1965; Oliveira et al., 1983; Kemper \& Rosenau, 1986), peneiramento úmido e turbidimetria (Pojasok \& Kay, 1990), resistência do agregado ao impacto de gotas (McCalla, 1944; Mazurak \& Mosher, 1968; Bruce-Okine \& Lal, 1975; Farres \& Cousen, 1985; Silva et al., 1995) e, mais recentemente, métodos envolvendo energia ultra-sônica, que permitem a quantificação da energia necessária para dispersão dos agregados (North, 1976; Gregorich et al., 1988; Raine \& So, 1993), têm sido utilizados para determinação da estabilidade de agregados do solo.

A escolha de um teste para determinação da estabilidade dos agregados de um solo depende da finalidade; portanto é importante distinguir entre os parâmetros testados, os efeitos dos métodos de preparação das amostras, e os efeitos dos próprios métodos (Matkin \& Smart, 1987). Em solos britânicos, os autores observaram que o peneiramento úmido se aplica a todos os tipos de solos, principalmente aos mais estáveis; para os menos estáveis e com argilas expansivas, citam um teste proposto por
Williams \& Cooke (1961) como o mais sensível para detectar diferenças. Segundo Farres \& Cousen (1985), a capacidade dos agregados em resistir ao impacto de gotas pode ser considerada o índice mais apropriado para representar a estabilidade do solo, uma vez que esse método compara-se, em parte, a processos que ocorrem no campo. Trabalhos usando simuladores de chuva e testes de impacto de gotas permitem identificar a relação entre a energia de impacto das gotas e a quebra dos agregados. Entretanto, o tempo e os custos limitam a adaptação destes testes em análises de rotina (Raine \& So, 1993).

O emprego da energia ultra-sônica na dispersão de amostras para avaliação da textura do solo tem sido difundido, sendo de uso mais recente na avaliação da estabilidade de agregados (Raine \& So, 1994), e existem alguns estudos sobre solos de clima temperado (North, 1976, 1979; Gregorich et al., 1988, 1989; Fuller \& Goh, 1992; Raine \& So, 1993; 1994; Tippkötter, 1994; Fuller et al., 1995). Raine \& So (1993) apresentaram resultados em curvas características de dispersão de solos, fornecendo indicativo do comportamento destes perante energias atuantes no campo, as quais são relativamente pequenas, quando comparadas à energia ultra-sônica requerida para completa dispersão de amostras. De acordo com North (1979), um índice de estabilidade de agregados que leve em conta a energia para dispersão total fornece poucas informações sobre o potencial que apresenta o manejo em causar a desagregação dos solos. O método de Raine \& So (1993) foi utilizado por Tippkötter (1994) na quantificação da energia necessária para dispersar agregados de diâmetro compreendido entre 0,06 e 2,00 mm, que, segundo o autor, pode ser indicativo daquela energia envolvida na erosão hídrica do solo. Para Eriksen et al. (1995), a estabilidade dos agregados aumenta com a redução do seu tamanho, sendo, portanto, necessário maior nível de energia para destruir microagregados comparativamente aos macroagregados. Gregorich et al. (1988) mostraram ser necessária energia de 300 a $500 \mathrm{~J} \mathrm{~mL}^{-1}$ para destruir agregados do tamanho areia, sendo que microagregados somente são destruídos após aplicados $1.500 \mathrm{~J} \mathrm{~mL}^{-1}$.

Objetivou-se, com este estudo, comparar diferentes métodos para a determinação da estabilidade de agregados em solos. 


\section{MATERIAL E MÉTODOS}

Foram utilizadas amostras de material de solo dos horizontes A e B de um Latossolo Roxo (LR) e uma Terra Roxa Estruturada (TR) da região de Lavras (MG). $\mathrm{Na}$ caracterização das amostras, os teores de carbono orgânico, $\mathrm{Fe}_{2} \mathrm{O}_{3}$ pelo ataque sulfúrico e granulometria pelo método da pipeta, foram determinados segundo Embrapa (1979); caulinita e gibbsita foram quantificadas na fração argila desferrificada, através de análise termo-diferencial (ATD). Na análise de estabilidade de agregados, as amostras coletadas foram secadas ao ar e cuidadosamente peneiradas, para obter agregados de diâmetro entre de 7,93 e 4,76 mm. Em todos os testes foi feito pré-umedecimento dos agregados em água por capilaridade, durante duas horas.

A estabilidade dos agregados foi determinada por peneiramento em água (PU), com um jogo de peneiras de 2, 1, 0,5, 0,25 e 0,105 mm, sendo determinados o diâmetro médio geométrico (DMG) e diâmetro médio ponderado (DMP), conforme Kemper \& Chepil (1965), e porcentagem de agregados maiores que $2 \mathrm{~mm}$. Cada amostra, em quatro repetições, apresentou um peso equivalente a $25 \mathrm{~g}$ de agregados secos em estufa $\left(105^{\circ} \mathrm{C}\right.$ por 24 horas).

A resistência dos agregados ao impacto de gotas foi medida segundo Angulo et al. (1984), com modificações que consistiram em passar cuidadosamente os agregados quebrados em cada teste, por um jogo de peneiras idêntico ao empregado no peneiramento úmido, considerando os agregados que permaneceram intactos após os testes (estáveis) como retidos na primeira peneira, empregados no cálculo de DMG, DMP, e porcentagem de agregados maiores que $2 \mathrm{~mm}$.

O tamanho das gotas foi avaliado pesando-se 100 gotas de água, e dividindo este por 100 para se obter o peso de cada gota. Considerando a densidade da água igual a $1 \mathrm{~g} \mathrm{~cm}^{-3}$, calculou-se o diâmetro da gota, assumindo-se forma esférica. Neste estudo, o diâmetro médio das gotas foi de $3,5 \mathrm{~mm}$, e caíram de uma altura arbitrária de $0,81 \mathrm{~m}$. $\mathrm{O}$ incremento da energia cinética desse tamanho de gotas, em queda a partir de $0,5 \mathrm{~m}$ ou mais, pode ser considerado desprezível (Silva et al., 1995). Na Fig. 1 é apresentado um esquema do gotejador usado neste estudo. A energia cinética aplicada foi calculada conforme equação proposta por Silva et al. (1995):

$\mathrm{EC}=\left[\Sigma\left(\Sigma\right.\right.$ gt $\left.\left.\mathrm{EC}_{\text {gota }}\right)\right] / \mathrm{M}$

onde:

EC é a energia cinética aplicada à amostra $\left(\mathrm{J} \mathrm{g}^{-1}\right)$;

gt é o número de gotas a que cada agregado resistiu até se romper;

$\mathrm{EC}_{\text {gota }}$ é a energia cinética de uma gota (J);

Mé a massa da amostra (20 agregados) em g.

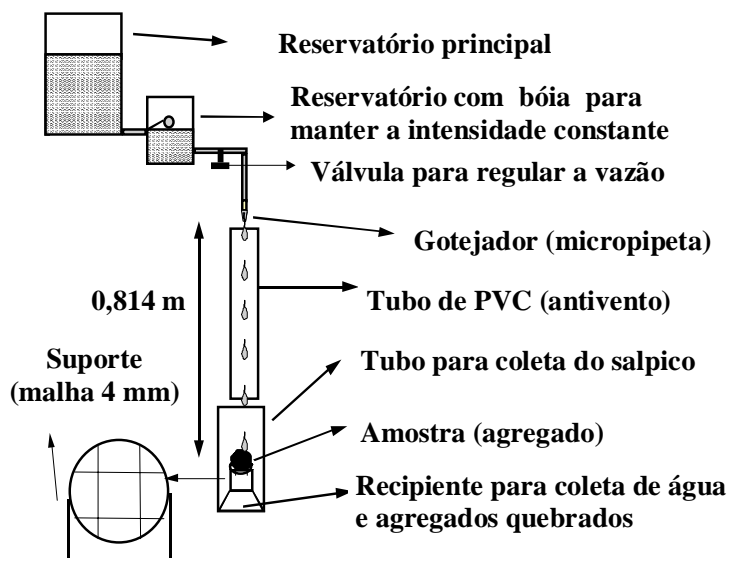

FIG. 1. Esquema do gotejador usado neste estudo para calcular a resistência ao impacto de gotas.

Foram realizados três testes de impacto de gotas. O primeiro (GT1), proposto por Angulo et al. (1984), com os agregados submetidos a, no máximo, 300 gotas, e intensidade de 120 gotas por minuto, avaliando-se o número de gotas para a quebra do agregado, considerando-se estáveis os agregados que resistirem as 300 gotas. No segundo (GT2), os agregados foram submetidos a 900 gotas, com intensidade 120 gotas por minuto, e no terceiro (GT3), foram submetidos a 300 gotas, com intensidade de 30 gotas por minuto. Cada amostra consistiu de 20 agregados escolhidos ao acaso, com quatro repetições. Após cada teste, os agregados retidos em cada peneira ( 2 a $0,105 \mathrm{~mm})$ foram secados em estufa $\left(105^{\circ} \mathrm{C}\right.$, por 24 horas), pesados, e os valores empregados no cálculo dos índices DMG, DMP e porcentagem de agregados maiores que $2 \mathrm{~mm}$.

No método de sonificação, foi utilizado um aparelho marca Misonix, modelo XL2020, operando a $20 \mathrm{kHz}$ no estágio 9 do mostrador, fornecendo uma potência de 192,50 W, correspondente a uma potência de $105,90 \pm 1,72 \mathrm{~W}$, calculada a partir do procedimento-padrão descrito por Sá (1998). Cada amostra, em quatro repetições, foi equivalente a $10 \mathrm{~g}$ de agregados secos. As sonificações foram feitas em béquer com $400 \mathrm{~mL}$ de água destilada, em uma relação solo:água de 1:40, durante $5,15,30,60,300,900,1.800$ e 2.700 segundos (US1, US2, US3, US4, US5, US6, US7 e US8, respectivamente). Estes valores correspondem às energias de 1,32, 3,97, 7,94, $15,88,79,42,238,27,476,53$ e $714,80 \mathrm{~J} \mathrm{~mL}^{-1}$, calculadas segundo Christensen (1985) pela equação:

$\mathrm{EA}=\left[\mathrm{P}_{\mathrm{c}} \mathrm{t}_{\mathrm{s}}\right] / \mathrm{v}$

onde:

EA é a energia ultra-sônica aplicada à amostra $\left(\mathrm{J} \mathrm{mL}^{-1}\right)$; 
$\mathrm{P}_{\mathrm{c}}$ é a potência (W) emitida pelo aparelho e calculada através de calibração (Sá, 1998);

$\mathrm{t}_{\mathrm{s}}$ é o tempo de sonificação (s);

$\mathrm{v}$ é o volume da suspensão (mL).

A ponta da haste do aparelho, com comprimento de $175 \mathrm{~mm}$ e diâmetro de $19 \mathrm{~mm}$, foi inserida na suspensão a uma profundidade de $85 \mathrm{~mm}$. Para reduzir o efeito da elevação de temperatura na amostra, o béquer foi colocado em banho de gelo durante a sonificação, e a temperatura da suspensão foi mantida abaixo de $40^{\circ} \mathrm{C}$, conforme sugerido por Gregorich et al. (1989). Em tempos longos de sonificação, as amostras eram resfriadas a cada 15 minutos, uma vez que ao final deste tempo, mesmo com banho de gelo, a temperatura se aproximava de $40^{\circ} \mathrm{C}$. Após cada sonificação, as amostras foram passadas em jogo de peneiras de 2, 1, 0,5, 0,25 e 0,105 mm. Em seguida, foram calculados, para cada tempo de sonificação, os índices DMG, DMP e porcentagem de agregados maiores que $2 \mathrm{~mm}$.

$\mathrm{O}$ estudo seguiu um esquema fatorial ( $2 \times 2 \times 12)$, em delineamento inteiramente casualizado, correspondendo, respectivamente, a 2 solos (LR e TR), 2 horizontes (A e B) e 12 métodos (PU, GT1, GT2, GT3, US1, US2, US3, US4, US5, US6, US7 e US8). A comparação entre solos e entre horizontes foi feita por meio da análise de variância (teste $\mathrm{F}$ a 5\%) e a comparação entre métodos foi feita com testes de médias (Tukey a 5\%) entre os respectivos índices (DMG, DMP e agregados maiores que $2 \mathrm{~mm}$ ) dentro de cada horizonte, assumindo que a energia aplicada às amostras foi equivalente nos diferentes métodos, quando não são encontradas diferenças entre os índices testados. Na Tabela 1, é apresentado o resumo da análise de variância e nas Tabelas 2 e 3, as propriedades químicas e físicas dos solos.

\section{RESULTADOS E DISCUSSÃO}

Nos dois solos estudados, em todos os métodos onde foram detectadas diferenças significativas, o horizonte A apresentou agregados mais estáveis (Tabela 4), devido ao seu teor de matéria orgânica mais elevado (Tabela 2), o que concorda com Tisdall \& Oades (1982), Roth et al. (1991) e Fuller et al. (1995). As diferenças entre os tratamentos GT2 e PU não foram significativas em relação ao Latossolo Roxo, nos índices DMP e agregados maiores que $2 \mathrm{~mm}$. Também não houve diferença significativa nos níveis mais altos de energia ultra-sônica, uma vez que, nestes casos, o material encontrava-se totalmente disperso. Nos dois solos, notadamente na Terra Roxa Estruturada, à medida que se aumenta o nível de energia ultra-sônica, relativamente, aumentam as diferenças entre os horizontes A e B, evidenciando o efeito benéfico da matéria orgânica também em níveis mais elevados de energia.

TABELA 1. Quadrado médio da análise de variância e coeficiente de variação (CV) do diâmetro médio geométrico (DMG), diâmetro médio ponderado (DMP) e porcentagem de agregados maiores que 2 mm (IE) para comparação dos métodos de determinação da estabilidade de agregados nos horizontes A e B de Latossolo Roxo e Terra Roxa Estruturada.

\begin{tabular}{|c|c|c|c|c|}
\hline \multirow[t]{2}{*}{ Fator de variação } & \multirow[t]{2}{*}{ GL } & \multicolumn{3}{|c|}{ Quadrado médio } \\
\hline & & DMG & DMP & $\mathrm{IE}$ \\
\hline Tratamento & 47 & $12,18 * *$ & $14,13 * *$ & $5769,81^{* * *}$ \\
\hline Método (M) & 11 & $42,17 * *$ & $54,25 * *$ & $21805,38 * *$ \\
\hline Solo (S) & 1 & $1,20 * *$ & $2,67 * *$ & $1579,76^{* *}$ \\
\hline Horizontes $(\mathrm{H})$ & 1 & $64,90 * *$ & $34,67 * *$ & $16821,77 * *$ \\
\hline$M \times S$ & 11 & $0,31 *$ & $0,21 * *$ & $96,67 * *$ \\
\hline $\mathrm{M} \times \mathrm{H}$ & 11 & $3,32 * *$ & $1,91 * *$ & $790,06 * *$ \\
\hline $\mathrm{S} \times \mathrm{H}$ & 1 & $0,20^{\mathrm{ns}}$ & $1,74 * *$ & $994,63 * *$ \\
\hline $\mathrm{M} \times \mathrm{S} \times \mathrm{H}$ & 11 & $0,21^{\mathrm{ns}}$ & $0,45^{* * *}$ & $197,43 * *$ \\
\hline Resíduo & 144 & 0,15 & 0,06 & 27,89 \\
\hline Total & 191 & & & \\
\hline $\mathrm{CV}(\%)$ & & 17,15 & 8,39 & 8,95 \\
\hline
\end{tabular}


TABELA 2. Teor de matéria orgânica (MO), óxidos de ferro $\left(\mathrm{Fe}_{2} \mathrm{O}_{3}\right.$, ataque por $\left.\mathrm{H}_{2} \mathrm{SO}_{4}\right)$, caulinita $(\mathrm{Ct})$, gibbsita (Gb) e energia cinética aplicada (EC) para os testes de impacto de gotas, em Latossolo Roxo (LR) e Terra Roxa Estruturada (TR).

\begin{tabular}{ccccccccc}
\hline Solo & Horizonte & $\mathrm{MO}$ & $\mathrm{Fe}_{2} \mathrm{O}_{3}$ & $\mathrm{Ct}$ & $\mathrm{Gb}$ & \multicolumn{3}{c}{ EC } \\
\cline { 6 - 8 } & & & & & & $\mathrm{GT} 1$ & $\mathrm{GT} 2$ & $\mathrm{GT3}$ \\
\hline \multirow{2}{*}{$\mathrm{LR}$} & $\mathrm{A}$ & 60 & 254,5 & - & - & 1,58 & 5,40 & 2,08 \\
& $\mathrm{~B}$ & 26 & 238,4 & 255,2 & 256,6 & 0,98 & 4,28 & 1,74 \\
$\mathrm{TR}$ & $\mathrm{A}$ & 31 & 254,2 & - & - & 0,97 & 4,03 & 1,01 \\
& $\mathrm{~B}$ & 12 & 237,7 & 254,0 & 25,2 & 0,42 & 1,73 & 0,48 \\
\hline
\end{tabular}

${ }^{1}$ GT1: 300 gotas, 120 gotas por minuto; GT2: 900 gotas, 120 gotas por minuto; GT3: 300 gotas, 30 gotas por minuto.

TABELA 3. Granulometria do Latossolo Roxo (LR) e Terra Roxa Estruturada (TR).

\begin{tabular}{|c|c|c|c|c|c|c|c|c|c|}
\hline \multirow[t]{2}{*}{ Solo } & \multirow[t]{2}{*}{ Horizonte } & \multirow[t]{2}{*}{ Argila } & \multirow[t]{2}{*}{ Silte } & \multirow[t]{2}{*}{ Areia } & \multicolumn{5}{|c|}{ Frações da areia } \\
\hline & & & & & Muito grossa & Grossa & Média & Fina & Muito fina \\
\hline & & & & & & $\left.\mathrm{g}^{-1}\right)-$ & & & \\
\hline \multirow[t]{2}{*}{ LR } & A & 710 & 120 & 170 & 20 & 40 & 40 & 30 & 40 \\
\hline & B & 670 & 190 & 140 & 20 & 20 & 30 & 30 & 40 \\
\hline \multirow[t]{2}{*}{$\mathrm{TR}$} & A & 440 & 320 & 240 & 30 & 30 & 30 & 60 & 90 \\
\hline & B & 700 & 190 & 110 & 10 & 10 & 10 & 40 & 40 \\
\hline
\end{tabular}

Na maioria dos métodos testados não foram encontradas diferenças significativas entre os solos (Tabela 4), embora os agregados do horizonte A do Latossolo Roxo tenham sido mais estáveis nos métodos PU e GT1, considerando-se o diâmetro médio geométrico. Com relação ao diâmetro médio ponderado, apenas os métodos US5 e US6 mostraram diferença significativa. Entretanto, observa-se que no método US5, os agregados do horizonte A da Terra Roxa Estruturada foram mais estáveis que no Latossolo Roxo. Talvez a qualidade da matéria orgânica, não avaliada no presente estudo, tenha influenciado este resultado para este nível de energia específico, o que precisa ser melhor verificado. Observa-se que métodos GT1 e US5 apresentaram diferenças significativas quanto á porcentagem de agregados maiores que $2 \mathrm{~mm}$, possivelmente devido aos maiores teores de matéria orgânica do horizonte A do Latossolo Roxo, mais importante na formação de macroagregados.

O horizonte B do Latossolo Roxo mostrou-se mais estável do que o da Terra Roxa Estruturada. Neste caso, o maior teor de gibbsita, e o teor elevado de óxidos de ferro (Tabela 2) podem conferir maior estabilidade (Lima et al., 1990; Veiga et al., 1993).
A diferença entre os resultados de estabilidade no horizonte B do LR foi maior à medida que se aumentou o nível de energia ultra-sônica aplicada, indicando que seus agregados tendem a resistir mais a este tipo de energia.

De maneira geral, não foi observado um padrão de comportamento entre solos e horizontes em função dos métodos testados (Tabela 4), e parâmetros avaliados, ou seja, não há uma mesma tendência nos resultados dos três métodos, exceto no método US4 (15,88 $\left.\mathrm{J} \mathrm{mL}^{-1}\right)$.

Quanto ao horizonte A do Latossolo Roxo (Tabela 4), o peneiramento úmido foi equivalente à aplicação de uma energia de 300 gotas $\left(1,58 \mathrm{~J} \mathrm{~g}^{-1}\right)$, com intensidade de 120 gotas por minuto e a uma energia ultra-sônica de 1,32 J mL-1. Os dados de energia, em cada teste de impacto de gotas, encontramse na Tabela 2. Os métodos GT2, GT3, US2 e US3 são equivalentes, ou seja, energias de 900 e 300 gotas $\left(5,40\right.$ e 2,08 $\left.\mathrm{J} \mathrm{g}^{-1}\right)$ a intensidades de 120 e 30 gotas por minuto, respectivamente, apresentam o mesmo efeito que 3,97 e 7,94 J mL-1 de energia ultra-sônica.

Pelos resultados de diâmetro médio geométrico (Tabela 4) verifica-se que os métodos PU, GT1, GT2, GT3, US1, US2 e US3 desagregaram menos o solo e 
TABELA 4. Comparação entre métodos para determinação da estabilidade de agregados pelo diâmetro médio geométrico (DMG), diâmetro médio ponderado (DMP) e porcentagem de agregados maiores que $2 \mathrm{~mm}$.

\begin{tabular}{|c|c|c|c|c|}
\hline \multirow[t]{2}{*}{ Método } & \multicolumn{2}{|c|}{ Latossolo Roxo } & \multicolumn{2}{|c|}{ Terra Roxa Estruturada } \\
\hline & Horizonte A & Horizonte B & Horizonte A & Horizonte B \\
\hline & \multicolumn{4}{|c|}{ DMG } \\
\hline PU & $4,82 \mathrm{aA} a$ & $3,96 \mathrm{bA} a$ & $4,24 \mathrm{aB} a$ & $2,90 \mathrm{bB} a b$ \\
\hline GT1 & $4,84 \mathrm{aA} a$ & $3,11 \mathrm{bA} b c$ & $4,09 \mathrm{aB} a$ & $3,39 \mathrm{bA} a$ \\
\hline GT2 & $3,84 \mathrm{aA} a b$ & $3,29 \mathrm{bA} b$ & $4,06 \mathrm{aA} a$ & $3,22 \mathrm{bA} a$ \\
\hline GT3 & $4,41 \mathrm{aA} a b$ & $3,22 \mathrm{bA} b$ & $4,28 \mathrm{aA} a$ & $3,37 \mathrm{bA} a$ \\
\hline US1 & $4,46 \mathrm{aA} a$ & $2,53 \mathrm{bAcd}$ & $4,32 \mathrm{aA} a$ & $2,08 \mathrm{bA} b c$ \\
\hline US2 & $4,03 \mathrm{aA} a b$ & 1,91bAde & $4,05 \mathrm{aA} a$ & $1,68 \mathrm{bAc}$ \\
\hline US3 & $3,88 \mathrm{aA} a b$ & $1,78 \mathrm{bA} e$ & $3,83 \mathrm{aA} a$ & $1,28 \mathrm{bAcd}$ \\
\hline US4 & $3,22 \mathrm{aA} b$ & $1,06 \mathrm{bA} f$ & $2,95 \mathrm{aA} b$ & $0,36 \mathrm{bB} d e$ \\
\hline US5 & $0,70 \mathrm{aB} c$ & $0,22 \mathrm{aAg}$ & $1,29 \mathrm{aAc}$ & $0,07 \mathrm{bA} e$ \\
\hline US6 & $0,12 \mathrm{aA} c$ & $0,07 \mathrm{aAg}$ & $0,06 \mathrm{aAd}$ & $0,06 \mathrm{aA} e$ \\
\hline US7 & $0,06 \mathrm{aA} c$ & $0,06 \mathrm{aAg}$ & $0,07 \mathrm{aAd}$ & $0,07 \mathrm{aA} e$ \\
\hline \multirow[t]{2}{*}{ US8 } & $0,06 \mathrm{aA} c$ & $0,06 \mathrm{aAg}$ & $0,06 \mathrm{aAd}$ & $0,06 \mathrm{aA} e$ \\
\hline & \multicolumn{4}{|c|}{ DMP } \\
\hline PU & $4,93 \mathrm{aA} a$ & $4,60 \mathrm{aA} a$ & $4,77 \mathrm{aA} a$ & $3,82 \mathrm{bB} a$ \\
\hline GT1 & $4,92 \mathrm{aA} a$ & $4,13 \mathrm{bA} b c$ & $4,59 \mathrm{aA} a$ & $4,20 \mathrm{bA} a$ \\
\hline GT2 & $4,57 \mathrm{aA} a b$ & $4,31 \mathrm{aA} a b$ & $4,67 \mathrm{aA} a$ & $4,07 \mathrm{bA} a$ \\
\hline GT3 & $4,83 \mathrm{aA} a b$ & $4,33 \mathrm{bA} a b$ & $4,74 \mathrm{aA} a$ & $4,16 \mathrm{bA} a$ \\
\hline US1 & $4,81 \mathrm{aA} a b$ & $3,96 \mathrm{bA} b c d$ & $4,77 \mathrm{aA} a$ & $3,51 \mathrm{bB} a b$ \\
\hline US2 & $4,68 \mathrm{aA} a b$ & $3,73 \mathrm{bAcd}$ & $4,67 \mathrm{aA} a$ & $3,38 \mathrm{bB} a b$ \\
\hline US3 & $4,62 \mathrm{aA} a b$ & $3,67 \mathrm{bAd}$ & $4,60 \mathrm{aA} a$ & $2,79 \mathrm{bB} b$ \\
\hline US4 & $4,41 \mathrm{aA} b$ & $3,05 \mathrm{bAe}$ & $4,31 \mathrm{aA} a$ & $1,80 \mathrm{bB} c$ \\
\hline US5 & $2,67 \mathrm{aB} c$ & $1,44 \mathrm{bA} f$ & $3,31 \mathrm{aA} b$ & $0,26 \mathrm{bB} d$ \\
\hline US6 & $0,84 \mathrm{aA} d$ & $0,19 \mathrm{bAg}$ & $0,15 \mathrm{aB} c$ & $0,15 \mathrm{aA} d$ \\
\hline US7 & $0,15 \mathrm{aA} e$ & $0,07 \mathrm{aAg}$ & $0,27 \mathrm{aA} c$ & $0,21 \mathrm{aAd}$ \\
\hline \multirow[t]{2}{*}{ US8 } & $0,08 \mathrm{aA} e$ & $0,08 \mathrm{aAg}$ & $0,11 \mathrm{aA} c$ & $0,11 \mathrm{aAd}$ \\
\hline & \multicolumn{4}{|c|}{ Agregados maiores que $2 \mathrm{~mm} \mathrm{( \% )}$} \\
\hline PU & $99,2 \mathrm{aA} a$ & $91,9 \mathrm{aA} a$ & $95,8 \mathrm{aA} a$ & $70,3 \mathrm{bB} a b$ \\
\hline GT1 & $98,9 \mathrm{aA} a$ & $80,5 \mathrm{bA} b c$ & $90,6 \mathrm{aB} a$ & $80,8 \mathrm{bA} a$ \\
\hline GT2 & $91,7 \mathrm{aA} a b$ & $85,1 \mathrm{aA} a b$ & $93,4 \mathrm{aA} a$ & $77,6 \mathrm{bB} a$ \\
\hline GT3 & $97,1 \mathrm{aA} a b$ & $86,0 \mathrm{bA} a b$ & $95,0 \mathrm{aA} a$ & $79,5 \mathrm{bA} a$ \\
\hline US1 & $96,5 \mathrm{aA} a b$ & $77,9 \mathrm{bA} b c$ & $95,6 \mathrm{aA} a$ & $65,8 \mathrm{bB} a b$ \\
\hline US2 & $93,6 \mathrm{aA} a b$ & $73,6 \mathrm{bAc}$ & $93,4 \mathrm{aA} a$ & $64,0 \mathrm{bB} a b$ \\
\hline US3 & $92,4 \mathrm{aA} a b$ & $72,3 \mathrm{bAc}$ & $92,0 \mathrm{aA} a$ & $52,7 \mathrm{bB} b c$ \\
\hline US4 & $88,1 \mathrm{aA} b$ & $59,7 \mathrm{bAd}$ & $85,8 \mathrm{aA} a$ & $33,5 \mathrm{bB} c$ \\
\hline US5 & $52,4 \mathrm{aB} c$ & $27,6 \mathrm{bA} e$ & $65,1 \mathrm{aA} b$ & $3,9 \mathrm{bB} d$ \\
\hline US6 & $15,5 \mathrm{aAd}$ & $2,3 \mathrm{bA} f$ & $1,4 \mathrm{aA} c$ & $1,7 \mathrm{aAd}$ \\
\hline US7 & $1,5 \mathrm{aA} e$ & $0,0 \mathrm{aA} f$ & $4,0 \mathrm{aA} c$ & $2,9 \mathrm{aA} d$ \\
\hline US8 & $0,0 \mathrm{aA} e$ & $0,1 \mathrm{aA} f$ & $0,6 \mathrm{aA} c$ & $1,0 \mathrm{aA} d$ \\
\hline
\end{tabular}

${ }^{1}$ Médias seguidas das mesmas letras não diferem entre si pelo teste $\mathrm{F}$ a $5 \%$ de probabilidade: letras minúsculas comparam os horizontes A e B dentro de cada solo; letras maiúsculas comparam os solos dentro de cada horizonte; letras em itálico comparam os métodos dentro de cada horizonte, dentro de cada solo, pelo teste de Tukey a $5 \%$.

2 PU: peneiramento úmido; GT1: 300 gotas a 120 gotas por minuto; GT2: 900 gotas a 120 gotas por minuto; GT3: 300 gotas a 30 gotas por minuto; US1, US2, US3, US4, US5, US6, US7, US8 correspondem respectivamente aos níveis de energia ultra-sônica de 1,32, 3,97, 7,94, 15,88, 79,42, 238,27, 476,53 e $714,80 \mathrm{~J} \mathrm{~mL}^{-1}$ 
foram equivalentes entre si, ou seja, o peneiramento úmido causou a mesma desagregação de 300, 900 e 300 gotas nas intensidades de 120, 120 e 30 gotas por minuto, respectivamente. $\mathrm{O}$ mesmo foi observado quanto a energias ultra-sônicas de 1,32 a 7,94 $\mathrm{J} \mathrm{mL}^{-1}$. Os níveis de energia em US6, US7 e US8 deixaram o solo totalmente disperso, não se constituindo em um bom parâmetro para avaliação da estabilidade de agregados (North, 1979; Tippkötter, 1994).

Os resultados de DMP e agregados maiores que $2 \mathrm{~mm}$ (Tabela 4) mostraram que os métodos PU, GT1, GT2, GT3, US1, US2, US3 e US4 foram os que menos desagregaram o horizonte A da Terra Roxa Estruturada, sem diferenças entre os mesmos. Neste caso, os níveis de energia do peneiramento úmido e dos testes de impacto de gotas podem ser considerados equivalentes às energias ultra-sônicas de 1,32 a $15,88 \mathrm{~J} \mathrm{~mL}^{-1}$.

Observa-se que, dentro de cada horizonte A, o DMP e porcentagens de agregados maiores que $2 \mathrm{~mm}$ apresentaram a mesma resposta aos métodos, enquanto o DMG mostrou um padrão de comportamento ligeiramente diferente. Angulo et al. (1984) observaram, quanto ao horizonte A de diversos solos, maior correlação entre a quantidade de agregados maiores que $2 \mathrm{~mm}$ com diâmetro médio ponderado em relação ao diâmetro médio geométrico. Segundo os autores, isto se deve ao uso de logaritmo no cálculo do diâmetro médio geométrico. Neste estudo, aparentemente, este fator logarítmico tornou este índice mais sensível para detectar diferenças entre os horizontes A. Esta tendência não foi observada com relação aos horizontes $\mathrm{B}$, onde a agregação é governada mais pela mineralogia do que pela matéria orgânica.

No que diz respeito ao horizonte B do Latossolo Roxo (Tabela 4), os métodos GT2 e GT3 mostraram resultados semelhantes, com valores baixos de desagregação, levando-se em conta o diâmetro médio geométrico. Já os métodos US5, US6, US7 e US8 apresentaram valores elevados de desagregação, com os dois últimos dispersando o solo por completo. O PU foi o método que causou menor desagregação, seguido pelos testes de impacto de gotas. A sonificação, mesmo em baixos níveis de energia, promoveu considerável desagregação deste horizonte. Quanto ao DMP, não houve diferença entre os métodos GT2 e GT3, apresentando baixa desagregação.
No horizonte B da Terra Roxa Estruturada (Tabela 4), os métodos US5, US6, US7 e US8 dispersaram completamente o solo. Com base no diâmetro médio geométrico, não foram encontradas diferenças significativas entre os testes de impacto de gotas. Quanto ao diâmetro médio ponderado, PU, GT1, GT2 e GT3 não se diferenciaram, e seus resultados ficaram próximos de US1 e US2. Já US3 e US4 diferiram dos demais. Ainda no mesmo horizonte, quanto à porcentagem de agregados maiores que $2 \mathrm{~mm}$, observa-se que testes de impacto de gotas não diferiram entre si, desagregando menos o solo, enquanto o peneiramento úmido promoveu a mesma desagregação que US1 e US2.

No horizonte B do Latossolo Roxo, comparandose todos os índices, o método do peneiramento úmido foi o que menos causou desagregação, seguido dos testes de impacto de gotas, e por fim os testes de sonificação, que, mesmo em baixos níveis de energia, desagregaram mais o solo do que os demais métodos (Tabela 4). Por outro lado, no horizonte B da Terra Roxa Estruturada, os métodos envolvendo impacto de gotas foram menos desagregantes, seguidos pelo peneiramento úmido e métodos ultrasônicos. Verifica-se, portanto, uma tendência de o horizonte B da Terra Roxa Estruturada ser mais susceptível à desagregação pelo peneiramento úmido, enquanto o horizonte B do Latossolo Roxo foi mais susceptível ao impacto de gotas. Possivelmente, o maior teor de gibbsita do Latossolo Roxo, associado aos maiores teores de óxidos de ferro (Tabela 2), que conferem estrutura mais granular, com unidades estruturais de menor tamanho com baixa coerência entre si, tenha tornado esse material mais susceptível ao salpicamento pelas gotas, o que concorda com Silva et al. (1995). Por outro lado, o horizonte B da Terra Roxa Estruturada, que apresenta estrutura em blocos subangulares bem definidos, reflexo de mineralogia mais caulinítica, apresentou maior resistência ao impacto das gotas

Foram feitas correlações entre índices obtidos por este método (DMG, DMP e agregados maiores que $2 \mathrm{~mm}$ ) e os mesmos obtidos por outros métodos (Tabela 5). De maneira geral, observam-se altos coeficientes de correlação com testes de impacto de gotas e ultra-som, principalmente US1, US2, US3 e US4, destacando-se o último, que apresentou coeficientes acima de 0,9 em todos os índices. Isso mos- 
TABELA 5. Coeficientes de correlação entre peneiramento umido (PU) e demais métodos testados, para os índices diâmetro médio geométrico (DMG), diâmetro médio ponderado (DMP) e porcentagem de agregados maiores que $2 \mathrm{~mm}$ (IE) para comparação dos métodos de determinação da estabilidade de agregados nos horizontes A e B de Latossolo Roxo e Terra Roxa Estruturada.

\begin{tabular}{cccc}
\hline Métodos $^{1}$ & DMG & DMP & IE \\
\hline GT1 & 0,77 & 0,69 & 0,71 \\
GT2 & 0,75 & 0,90 & 0,93 \\
GT3 & 0,76 & 0,89 & 0,92 \\
US1 & 0,88 & 0,91 & 0,91 \\
US2 & 0,83 & 0,87 & 0,88 \\
US3 & 0,87 & 0,95 & 0,95 \\
US4 & 0,91 & 0,96 & 0,95 \\
US5 & 0,63 & 0,89 & 0,89 \\
US6 & 0,75 & 0,55 & 0,51 \\
US7 & 0,12 & $-0,15$ & $-0,21$ \\
US8 & 0,78 & $-0,71$ & $-0,77$ \\
\hline
\end{tabular}

GT1: 300 gotas, 120 gotas por minuto; GT2: 900 gotas, 120 gotas por minuto; GT3: 300 gotas, 30 gotas por minuto; US1, US2, US3,US4, US5, US6, US7 e US8 correspondem às energias de 1,32, 3,97, 7,94 $15,88,79,42,238,27,476,53$ e $714,80 \mathrm{~J} \mathrm{~mL}^{-1}$, respectivamente.

tra que US4 pode ser um bom parâmetro para avaliação da estabilidade de agregados, uma vez que a energia aplicada é definida $\left(15,88 \mathrm{~J} \mathrm{~mL}^{-1}\right)$, ao contrário do que ocorre com o peneiramento úmido que, embora prático, o nível de energia aplicado é desconhecido, o que concorda com Fuller \& Goh (1992). A energia também pode ser quantificada em testes de impacto de gotas, embora estes sejam mais trabalhosos que testes envolvendo ultra-som. Quanto aos testes US5, US6, US7 e US8, apresentaram coeficientes de correlação mais reduzidos, alguns até negativos, o que pode ser atribuído aos altos níveis de energia aplicados, que dispersaram completamente o solo, e portanto, não constituem bom parâmetro para avaliação da estabilidade de agregados, o que está em concordância com North (1979) e Tippkötter (1994).

Os resultados deste estudo evidenciam que a estabilidade de agregados depende não só das características de cada solo, mas também da natureza das forças disruptivas aplicadas, fazendo com que diferenças na estabilidade de agregados possam ser devidas a diferenças em metodologias, quanto ao tipo e quantidade de energia aplicada, conforme sugerido por Fuller \& Goh (1992) e Beare \& Bruce (1993).

\section{CONCLUSÕES}

1. O Latossolo Roxo apresenta a maior estabilidade de agregados.

2. Todos os métodos utilizados mostram que os agregados do horizonte $\mathrm{A}$ são mais resistentes que o horizonte $\mathrm{B}$.

3. O horizonte B do Latossolo Roxo é resistente ao peneiramento úmido, enquanto o horizonte $\mathrm{B}$ da Terra Roxa Estruturada é mais resistente ao impacto de gotas.

4. Diâmetro médio ponderado e agregados maiores que $2 \mathrm{~mm}$ apresentam comportamentos semelhantes no horizonte A de ambos os solos estudados.

5. Energia ultra-sônica de 15,88 $\mathrm{J} \mathrm{mL}^{-1}$ mostra-se mais sensível em detectar diferenças entre os solos estudados.

\section{REFERÊNCIAS}

ANGULO, J.R. Relações entre a erodibilidade e algumas propriedades de solos brasileiros. Curitiba : UFRPR, 1983. 154p. Dissertação de Mestrado.

ANGULO, R.J.; ROLOFF, G.; SOUZA, M.L.P. Correlação entre diferentes formas de determinação e representação da estabilidade e resistência dos agregados do solo. Revista Brasileira de Ciência do Solo, Campinas, v.8, n.1, p.7-12, jan./abr. 1984.

BEARE, M.H.; BRUCE, R.R. A comparison of methods for measuring water-stable aggregates: implications for determining environmental effects on soil structure. Geoderma, Amsterdam, v.56, n.1, p.87104, Mar. 1993

BRUCE-OKINE, E.; LAL, R. Soil erodibility as determined by raindrop technique. Soil Science, Baltimore, v.119, n.2, p.149-157, Feb. 1975.

CHRISTENSEN, B.T. Carbon and nitrogen in particle size fractions isolated from Danish arable soils by ultrasonic dispersion and gravity sedimentation. Acta Agriculturae Scandinavica, Section B, Oslo, v.35, n.2, p.175-187, 1985. 
DERPSCH, R.; ROTH, C.H.; SIDIRAS, N.; KÖPKE, U. Controle da erosão no Paraná, Brasil: sistemas de cobertura do solo, plantio direto e preparo conservacionista do solo. Eschborn : Gesellschaft für Technische Zusammenarbeit/IAPAR, 1991. 260p.

EGASHIRA, K.; KAETSU, Y.; TAKUMA, K. Aggregate stability as an index of erodibility of Andosoils. Soil Science and Plant Nutrition, Tokyo, v.29, n.4, p.473-481, 1983.

EMBRAPA. Serviço Nacional de Levantamento e Conservação de Solo (Rio de Janeiro, RJ). Manual de métodos de análise de solo. Rio de Janeiro, 1979. não paginado.

ERIKSEN, J.; LEFROY, R.D.B.; BLAIR, G.J. Physical protection of soil organics studied using acetylacetone extraction at various intensities of ultrasonic dispersion. Soil Biology \& Biochemistry, Oxford, v.27, n.8, p.1005-1010, Aug. 1995.

FARRES, P.J.; COUSEN, S.M. An improved method of aggregate stability measurement. Earth Surface Processes and Landforms, Chichester, v.10, p.321329, 1985.

FULLER, L.G.; GOH, T.G. Stability-energy relationships and their application to aggregation studies. Canadian Journal of Soil Science, Ottawa, v.72, n.4, p.453-466, Nov. 1992.

FULLER, L.G.; GOH, T.G.; OSCARSON, D.W. Cultivation effects on dispersive clay of soil aggregates. Canadian Journal of Soil Science, Ottawa, v.75, n.1, p.101-107, Feb. 1995.

GREGORICH, E.G.; KACHANOSKI, R.G.; VORONEY, R.P. Carbon mineralization in soil size fractions after various amounts of aggregate disruption. Journal of Soil Science, Oxford, v.40, n.3, p.649-659, Sept. 1989.

GREGORICH, E.G.; KACHANOSKI, R.G.; VORONEY, R.P. Ultrasonic dispersion of aggregates: Distribution of organic matter in size fractions. Canadian Journal of Soil Science, Ottawa, v.68, n.2 p.395-403, May 1988.

KEMPER, W.D.; CHEPIL, W.S. Size distribution of aggregates. In: BLACK, C.A. (Ed.). Methods of soil analysis. Madison : American Society of Agronomy, 1965. v.1, p.499-509.
KEMPER, W.D.; ROSENAU, R.C. Aggregate stability and size distribution. In: KLUTE, A. (Ed.). Methods of soil analysis. Madison : American Society of Agronomy, 1986. v.1, p.425-442.

LIMA, J.M.; CURI, N.; RESENDE, M.; SANTANA, D.P. Dispersão do material de solo em água para avaliação indireta da erodibilidade de latossolos. Revista Brasileira de Ciência do Solo, Campinas, v.14, n.1, p.85-90, jan./abr. 1990.

McCALLA, T.M. Water drop method of determining the stability of soil structure. Soil Science Society of America. Proceedings, Madison, v.7, p.209-214, 1944.

MATKIN, E.A.; SMART, P. A comparison of tests of soil structural stability. Journal of Soil Science, Oxford, v.38, n.1, p.123-135, Mar. 1987.

MAZURAK, A.P.; MOSHER, P.N. Detachment of soil aggregates by simulated rainfall. Soil Science Society of America. Proceedings, Madison, v.34, n.5, p.798-800, Sept./Oct. 1970.

MAZURAK, A.P.; MOSHER, P.N. Detachment of soil particles in simulated rainfall. Soil Science Society of America. Proceedings, Madison, v.32, n.5 p.716719, Sept./Oct. 1968.

NORTH, P.F. Assessment of the ultrasonic method of determining soil structural stability in relation to soil management properties. Journal of Soil Science, Oxford, v.30, n.3, p.463-472, Sept. 1979

NORTH, P.F. Towards an absolute measurement of soil structural stability using ultrasound. Journal of Soil Science, Oxford, v.27, n.4, p.451-459, Dec. 1976.

OLIVEIRA, M.; CURI, N.; FREIRE, J.C. Influência do cultivo na agregação de um podzólico vermelho-amarelo textura média/argilosa da região de Lavras, MG. Revista Brasileira de Ciência do Solo, Campinas, v.7, n.3, p.317-322, set./dez. 1983.

POJASOK, T.; KAY, B.D. Assessment of a combination of wet sieving and turbidimetry to characterize the structural stability of moist aggregates. Canadian Journal of Soil Science, Ottawa, v.70, n.1, p.3342, Feb. 1990.

RAINE, S.R.; SO, B. An energy based parameter for the assessment of aggregate bond energy. Journal of Soil Science, Oxford, v.44, n.2, p.249-259, June 1993. 
RAINE, S.R.; SO, B. Ultrasonic dispersion of soil in water: the effect of suspension properties on energy dissipation and soil dispersion. Australian Journal of Soil Research, Collingwood, v.32, n.6, p.11571174, 1994

REICHERT, J.M.; VEIGA, M. da; CABEDA, M.S.V. Índices de estabilidade de agregados e suas relações com características e parâmetros de solo. Revista Brasileira de Ciência do Solo, Campinas, v.17, n.2, p.283-290, maio/ago. 1993.

ROTH, C.H.; CASTRO FILHO, C. de; MEDEIROS, G.B de. Análise de fatores físicos e químicos relacionados com a agregação de um Latossolo Roxo distrófico. Revista Brasileira de Ciência do Solo, Campinas, v.15, n.3, p.241-248, set./dez. 1991.

SÁ, M.A.C. de. Quantificação da energia para dispersão dos agregados de Latossolo Roxo e Terra Roxa Estruturada. Lavras : UFLA, 1998. 92p. Dissertação de Mestrado.

SANTOS, D. Perdas de solo e produtividade de pastagens nativas melhoradas sob diferentes práticas de manejo em Cambissolo distrófico (Epiálico) dos campos da Mantiqueira, MG. Lavras : ESAL, 1993. 99p. Dissertação de Mestrado.

SILVA, M.L.N. Erodibilidade e impacto direto de gotas de chuva simulada sobre a superfície de latossolos sob cerrado. Lavras : ESAL, 1994. 109p. Dissertação de Mestrado.
SILVA, M.L.N.; CURI, N.; MARQUES, J.J.G.S.M.; FERREIRA, M.M; LIMA, L.A. Resistência ao salpico provocado por impacto de gotas de chuva simulada em latossolos e sua relação com características químicas e mineralógicas. Ciência e Prática, Lavras, v.19, n.2, p.348-354, abr./jun. 1995.

SUN, H.; LARNEY, F.J.; BULLOCK, M.S. Soil amendments and water-stable aggregation of a desurfaced Dark Brown Chernozem. Canadian Journal of Soil Science, Ottawa, v.75, n.3, p.319325, Aug. 1995.

TIPPKÖTTER, R. The effect of ultrasound on the stability of mesoaggregates $(60-200 \mu \mathrm{m})$. Zeitschrift fuer Pflanzenernährung und Bodenkunde, Weinheim, v.157, n.2, p.99-104, Apr. 1994.

TISDALL, J.M.; OADES, J.M. Organic matter and waterstable aggregates in soils. Journal of Soil Science, Oxford, v.33, n.1, p.141-163, Mar. 1982.

VEIGA, M. da; CABEDA, M.S.V.; REICHERT, J.M. Erodibilidade em entressulcos de solos do Rio Grande do Sul. Revista Brasileira de Ciência do Solo, Campinas, v.17, n.1, p.121-128, jan./abr. 1993.

WILLIAMS, R.J.B.; COOKE, G.W. Some effects of farmyard manure and of grass residues on soil structure. Soil Science, Baltimore, v.92, n.1, p.3039, Jan. 1961.

YODER, R.E. A direct method of aggregate analysis of soils and a study of the physical nature of erosion. American Society of Agronomy Journal, Madison, v.28, p.337-351, 1936. 